\title{
Restricted Boltzmann Machines for Quantum States with Non-Abelian or Anyonic Symmetries
}

\author{
Tom Vieijra, ${ }^{1,}$ Corneel Casert, ${ }^{1}$ Jannes Nys, ${ }^{1}$ Wesley De Neve,,${ }^{2,3}$ Jutho Haegeman, ${ }^{1}$ \\ Jan Ryckebusch, ${ }^{1}$ and Frank Verstraete ${ }^{1}$ \\ ${ }^{1}$ Department of Physics and Astronomy, Ghent University, B-9000 Ghent, Belgium \\ ${ }^{2}$ Center for Biotech Data Science, Ghent University Global Campus, 21985 Incheon, Republic of Korea \\ ${ }^{3} I D L a b$, Department of Electronics and Information Systems, Ghent University, B-9052 Ghent, Belgium
}

(Received 28 May 2019; accepted 11 February 2020; published 2 March 2020)

\begin{abstract}
Although artificial neural networks have recently been proven to provide a promising new framework for constructing quantum many-body wave functions, the parametrization of a quantum wave function with non-abelian symmetries in terms of a Boltzmann machine inherently leads to biased results due to the basis dependence. We demonstrate that this problem can be overcome by sampling in the basis of irreducible representations instead of spins, for which the corresponding ansatz respects the non-abelian symmetries of the system. We apply our methodology to find the ground states of the one-dimensional antiferromagnetic Heisenberg (AFH) model with spin-1/2 and spin-1 degrees of freedom, and obtain a substantially higher accuracy than when using the $s_{z}$ basis as an input to the neural network. The proposed ansatz can target excited states, which is illustrated by calculating the energy gap of the AFH model. We also generalize the framework to the case of anyonic spin chains.
\end{abstract}

DOI: 10.1103/PhysRevLett.124.097201

Introduction.-Driven by the rapidly advancing research in artificial intelligence, many-body physics has embraced machine learning (ML) as a powerful tool to tackle nontrivial problems [1]. Applications include the use of neural networks for phase classification [2-10], accelerating Monte Carlo algorithms [11-15], and ML-based generative modeling of distributions in many-body physics [16-19]. The connection between the renormalization group and (deep) learning has also been highlighted [20-23].

Quantum mechanical spin systems play a key role in the field of many-body physics. In Ref. [19], a particular class of artificial neural networks (ANN), namely restricted Boltzmann machines (RBM), is introduced as a variational ansatz for the wave functions of many-body spin systems. The versatility of the ANN ansatz has been illustrated in studies of bosonic systems [24,25], (chiral) topological states [26-28], frustrated systems [29,30], and open systems [31-34]. The RBM ansatz has been studied from the perspective of entanglement [35], and was shown to embody volume-law entanglement. In this light, the connections and differences between RBMs and matrix product states have been laid out [36,37].

The invariance of wave functions under symmetries, in particular $\mathrm{SU}(2)$ symmetry, is important for applications such as quantum chemistry $[38,39]$ and the description of spin liquids $[40,41]$. In quantum chemistry, wave functions are eigenfunctions of the total angular momentum operators $\widehat{J}^{2}$ and $\widehat{J}_{z}$, and spin liquid states do not break any symmetry of the Hamiltonian. The capacity of RBMs to capture long-range correlations and their independence of the problem's geometry make them a prime candidate for these applications.

In this Letter, we introduce a methodology for constructing an RBM variational wave function that transforms as an irreducible representation of $\mathrm{SU}(2)$. This wave function is designed to have a well-defined total angular momentum. Hence the method provides direct access to the construction of excited states. The challenge of imposing physical symmetries on the ANN ansatz has been addressed for finite Abelian symmetry groups [42], but this approach is not directly applicable to non-abelian symmetries. The proposed ansatz is not restricted to SU(2) symmetry and can be applied to other non-abelian symmetries, as well as anyonic spin chains.

RBM wave functions.-We use RBMs, which are energybased generative neural networks, as a variational ansatz for quantum many-body spin systems. In the context of this work, the RBM models a distribution $\Psi\left(x_{1}, \ldots, x_{N}\right) \equiv$ $\Psi(\mathbf{x})$ of the variables $x_{i \in\{1, \ldots, N\}}$, characterized by the energy function

$$
E_{\mathrm{RBM}}(\mathbf{x}, \mathbf{h} ; \mathcal{W})=\sum_{i}^{N} \sum_{j}^{M} w_{i j} x_{i} h_{j}+\sum_{i}^{N} a_{i} x_{i}+\sum_{j}^{M} b_{j} h_{j} .
$$

Here, $h_{j \in\{1, \ldots, M\}} \in\{-1,1\}$ is a set of binary latent variables. The set $\mathcal{W}=\left\{w_{i j}, a_{i}, b_{j}\right\}$ are variational parameters: $w_{i j}$ are the weights connecting variables $x_{i}$ and $h_{j}$, 
and $a_{i}\left(b_{j}\right)$ are the biases of the physical (latent) variables $x_{i}\left(h_{j}\right)$. The ratio of the number of latent variables $M$ and the number of physical variables $N$, defined by $\alpha \equiv M / N$, is a measure of the complexity of the model.

The RBM was introduced in Ref. [19] as a variational ansatz for quantum many-body wave functions by modeling the probability amplitudes $\Psi(\mathbf{x})$ of the wave function $|\Psi\rangle=\sum_{\mathbf{x}} \Psi(\mathbf{x})|\mathbf{x}\rangle$ as the marginalized Boltzmann distribution

$$
\Psi(\mathbf{x} ; \mathcal{W}) \equiv \sum_{\mathbf{h}} e^{-E_{\mathrm{RBM}}(\mathbf{x}, \mathbf{h} ; \mathcal{W})}, \quad \mathcal{W} \subset \mathbb{C} .
$$

This model is an extension of the RBM that is used to represent classical probability distributions as a marginalization over hidden variables of a Boltzmann distribution. In this work, $x_{i}$ is not necessarily a binary variable but remains discrete. We therefore replace $w_{i j} x_{i}$ with a set of general discrete functions $w_{i j}\left[x_{i}\right]$. This has the effect of transforming the variables $x_{i}$ nonlinearly before constructing the RBM energy function of Eq. (1).

To find eigenstates of a Hamiltonian $\widehat{H}$, we use the variational principle to minimize the energy functional

$$
E(\Psi ; \mathcal{W})=\frac{\langle\Psi|\widehat{H}| \Psi\rangle}{\langle\Psi \mid \Psi\rangle}
$$

with respect to the parameters $\mathcal{W}$, for which we use the stochastic reconfiguration method [43].

$S U(2)$ symmetry.-Hamiltonians with spin-rotation symmetry are omnipresent throughout the various domains of quantum many-body physics. Examples of SU(2)symmetric quantum-mechanical spin chains are the antiferromagnetic Heisenberg model (AFH), the bilinearbiquadratic model, and the Majumdar-Ghosh model. In order to illustrate the potential of the proposed methodology, we focus on the AFH Hamiltonian

$$
\widehat{H}_{\mathrm{AFH}}=\sum_{i=1}^{N-1} \hat{\mathbf{s}}_{i} \cdot \hat{\mathbf{s}}_{i+1},
$$

with spin-1/2 and spin-1 degrees of freedom in one dimension, which we study with open boundary conditions.

The main contribution of this work is the construction of a method to include $\mathrm{SU}(2)$ symmetry in ANN wave functions. This procedure yields wave functions $\left|J M_{J}\right\rangle$ with well-defined total angular momentum $J$ and projection $M_{J}$. These wave functions belong to the subspace spanned by states with quantum numbers $J$ and $M_{J}$ of the full Hilbert space of the system. Part of the energy spectrum of the system can be uncovered by finding the variational minima in the subspaces with fixed quantum numbers $\left|J M_{J}\right\rangle$. For example, the gap of the AFH can be calculated after constructing the ground state $\left|J=0 M_{J}=0\right\rangle$ and the first excited state $\left|J=1 M_{J} \in\{-1,0,1\}\right\rangle$.

To construct wave functions $\left|J M_{J}\right\rangle$, we use ClebschGordan coefficients to couple two spins, $\hat{\mathbf{s}}_{1}$ and $\hat{\mathbf{s}}_{2}$, to a single angular momentum degree of freedom $\hat{\mathbf{j}}_{2}=\hat{\mathbf{s}}_{1}+\hat{\mathbf{s}}_{2}$

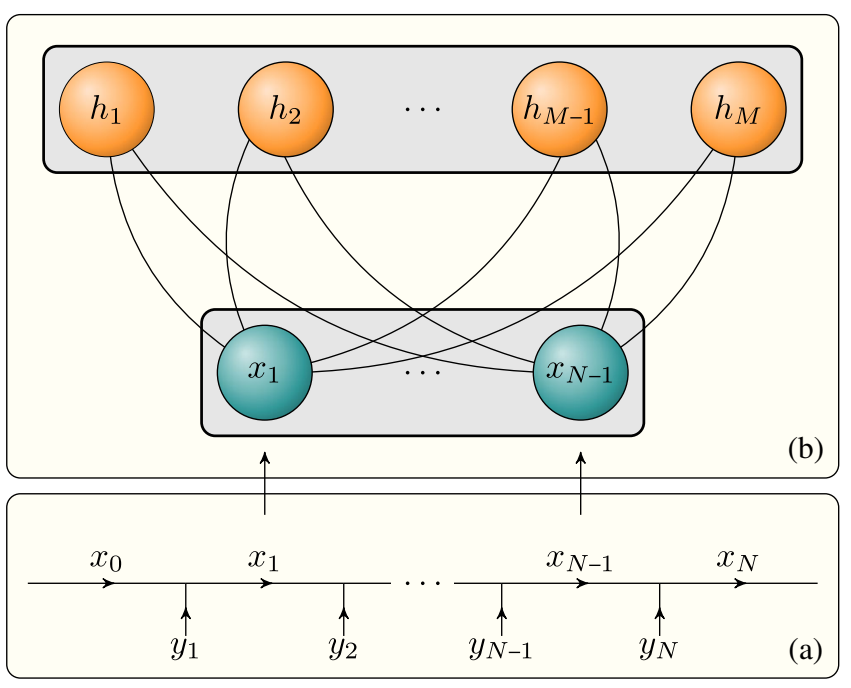

FIG. 1. Graphical representation of the variational wave function proposed in this work. (a) A basis in which all the degrees of freedom $y_{i \in\{1, \ldots, N\}}$ are coupled is proposed. The resulting intermediate degrees of freedom $x_{i \in\{1, \ldots, N-1\}}$ act as the input of the RBM, as represented in (b). The RBM defines the expansion coefficients of the wave function in this basis. The optimal coefficients minimize the energy functional of Eq. (3).

$\left|s_{1} s_{2} ; j_{2} m_{j_{2}}\right\rangle=\sum_{m_{s_{1}}, m_{s_{2}}}\left\langle s_{1} m_{s_{1}}, s_{2} m_{s_{2}} \mid j_{2} m_{j_{2}}\right\rangle\left|s_{1} m_{s_{1}}, s_{2} m_{s_{2}}\right\rangle$.

Given a system of $N$ spins $\hat{\mathbf{s}}_{i \in\{1, \ldots, N\}}$, we construct states with total angular momentum $J$ by starting at the left side of the chain and using Eq. (5) to couple the first two spins to the angular momentum $j_{2}$. Next, $j_{2}$ is coupled to $s_{3}$. This process is repeated till reaching the end of the chain, resulting in a total angular momentum $J$. This is depicted in Fig. 1(a) with $y_{i} \equiv s_{i}, x_{i} \equiv j_{i}$ and $x_{0}=0, x_{N}=J$. Given the large amount of intermediate couplings, it is nontrivial and numerically challenging to transform the wave function in the coupled basis back into the $\hat{\mathbf{s}}_{i \in\{1, \ldots, N\}}$ basis. As will become clear, however, observables of the studied quantum systems can be reliably and efficiently computed in the basis of coupled angular momenta.

In the ansatz proposed in Ref. [19], the spin projections $m_{s_{i}}$ of $\hat{\mathbf{s}}_{i}$ are used as input values for the RBM in Eq. (2). Rather, we use the intermediate degrees of freedom $j_{k}$ as input [Fig. 1(b)], which produces the wave function

$$
|\Psi\rangle=\sum_{j_{k}} \Psi\left(j_{1}, \ldots, j_{N-1}\right)\left|j_{1}, \ldots, j_{N-1} ; J M_{J}\right\rangle,
$$

where $\sum_{j_{k}}$ denotes a summation over all physically allowed configurations $j_{k \in\{1, \ldots, N-1\}}$. Equation (6) transforms as an irreducible representation of SU(2), labeled by total angular momentum $J$, with dimension $2 J+1$. For the states with $J=0$ (of which the ground state of the spin-1/2 $\mathrm{AFH}$ is an example), the state is manifestly invariant under 
$\mathrm{SU}(2)$ transformations, as the irreducible representation has one dimension. More information on this basis transformation can be found in the Supplemental Material [44]. The above procedure can be readily extended to other non-abelian symmetries by decomposing the degrees of freedom in irreducible representations of the symmetry group and finding the equivalent of the Clebsch-Gordan coefficients in Eq. (5) to relate the irreducible representation of a system to those of its subsystems.

Spin-1/2 AFH.-Finding the ground state of the spin$1 / 2 \mathrm{AFH}$ in the coupled spin basis amounts to variationally minimizing the energy functional of Eq. (3) in the subspace defined by total angular momentum $J=0$. The accuracy of the variational wave function can be assessed by comparison to energies obtained with exact diagonalization (ED) $\Delta E_{0}=\left|\left(E_{0}-E_{0, \text { exact }}\right) / E_{0 \text {,exact }}\right|$, the magnitude of the variance of the Hamiltonian $\operatorname{Var}(\widehat{H})=\left\langle\widehat{H}^{2}\right\rangle-\langle\widehat{H}\rangle^{2}$, and the weight $\varepsilon$ of excited states in the wave function. The latter can be found by writing $|\Psi\rangle=\sqrt{1-\varepsilon^{2}}\left|\Psi_{\mathrm{GS}}\right\rangle+\varepsilon\left|\Psi_{\perp}\right\rangle$, where $\left|\Psi_{\mathrm{GS}}\right\rangle$ is the exact ground state wave function and $\left|\Psi_{\perp}\right\rangle$ is a normalized superposition of states perpendicular to $\left|\Psi_{\mathrm{GS}}\right\rangle$. The parameter $\varepsilon$ is a measure for the accuracy of the variational ground state as it measures the spurious content in $|\Psi\rangle$. An upper bound on $\varepsilon$ is given by the relation $\operatorname{Var}(\widehat{H}) \geq \varepsilon^{2} G^{2}$, with $G \equiv E_{1}-E_{0}$ the difference between the energies of the first excited state and the ground state.

In Figs. 2(a)-2(c), we compare these convergence criteria for the ground states obtained with the RBM ansatz in the coupled basis and in the $s_{z}$ basis, as a function of the ratio $\alpha$. Both the relative energy error and $\operatorname{Var}(\widehat{H})$ are systematically lower when using the coupled basis compared to the $s_{z}$ basis. For small systems, where ED is feasible, the parameter $\varepsilon$ can be determined exactly by computing the overlap $\left\langle\Psi_{\mathrm{GS}} \mid \Psi\right\rangle=\sqrt{1-\varepsilon}$. We obtain consistently lower values of $\varepsilon$ in the coupled basis. Related to this, we see that the spin-spin correlators are consistently better described, and inherently unbiased, in the coupled basis. This is described in the Supplemental Material [44].

Also with the eye on gaining profound insight in the structure of the wave function, our methodology offers opportunities by studying the weight of the expansion coefficients in the ansatz of Eq. (6). We find that the basis state with the largest modulus has all pairs of neighboring spins coupled to a singlet. Next in importance are states with two neighboring triplets coupled to a singlet, on a background of singlets. More information on the structure of the wave function can be found in the Supplemental Material [44].

The introduction of the coupled basis allows us to find the variational minimum of the energy functional of Eq. (3) in a subspace with specific $\left|J M_{J}\right\rangle$, which enables us to construct excited states. We demonstrate this by calculating the energy difference between the lowest lying eigenstate in the subspaces defined by $\left|J=1 M_{J}=0\right\rangle$ and $\left|J=0 M_{J}=0\right\rangle$. As the AFH is critical, the gap $G$ vanishes

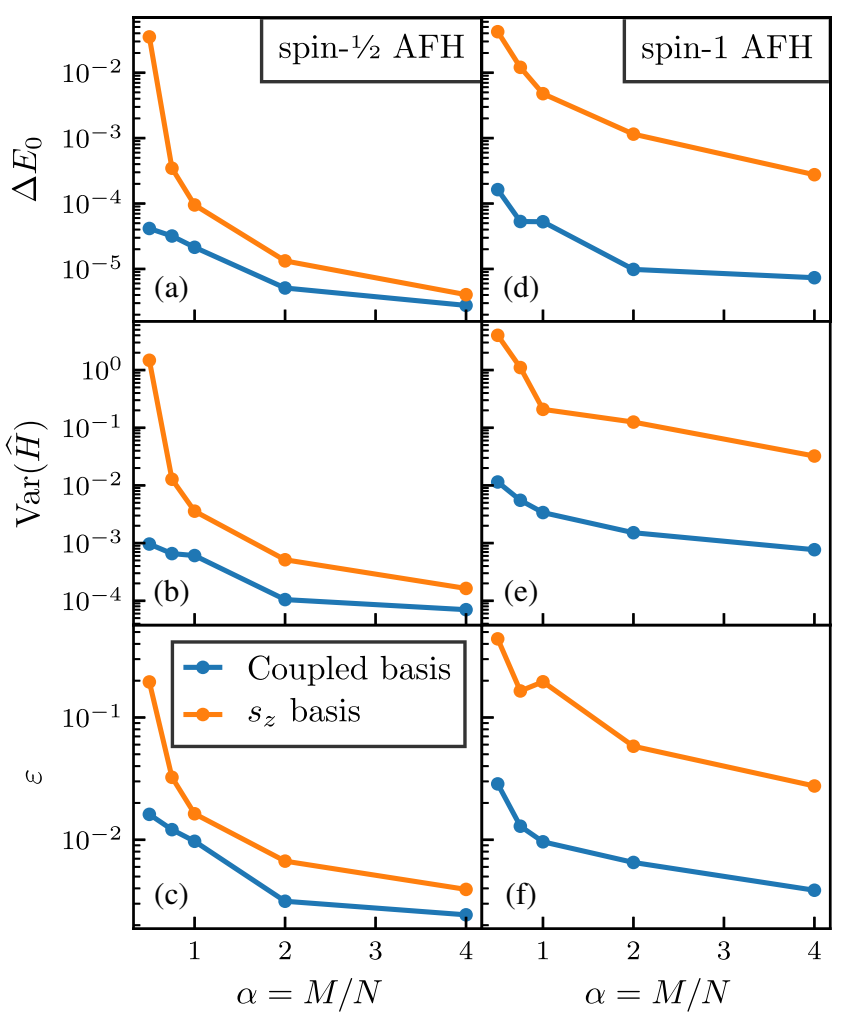

FIG. 2. Convergence of the ground-state properties as a function of the ratio $\alpha$ of hidden to visible units, for the spin- $1 / 2$ (left column, system size $L=22$ ), and spin-1 (right column, $L=12$ ) AFH. Top panels: energy error relative to the exact ground state energy. Middle panels: variance of the Hamiltonian. Bottom panels: content $\varepsilon$ of excited states in the ground-state approximation. The used hyperparameters can be found in the Supplemental Material [44].

as $G \propto N^{-1}$ for $N \rightarrow \infty$. The gap as a function of system size is depicted in Fig. 3(a) and matches results obtained with ED or density matrix renormalization group (DMRG). The relative energy errors $\Delta E_{0}$ on the ground state range from $\mathcal{O}\left(10^{-5}\right)$ for the smallest system sizes to $\mathcal{O}\left(10^{-4}\right)$ for larger systems. The errors on the excited state energies $\Delta E_{1}$ are generally slightly larger.

Spin-1 AFH.-Physically, the spin-1 AFH is inherently different from the spin-1/2 AFH. Whereas the spin- $1 / 2$ AFH is gapless in the thermodynamic limit, the spin-1 AFH has a fourfold degenerate ground state (consisting of a spin singlet and a spin triplet), above which a gap exists. The degeneracy of the ground state arises from the presence of effective spin- $1 / 2$ degrees of freedom at the edges of the system. The interaction between these effective degrees of freedom is exponentially suppressed with system size, resulting in two free spin- $1 / 2$ degrees of freedom in the thermodynamic limit [48]. For finite systems, the ground state is nondegenerate and a spin singlet, in accordance with Marshall's theorem [49]. The physical differences between the spin- $1 / 2$ and spin- 1 AFH make it interesting to investigate the representational ability of RBMs in both cases. Figures $2(\mathrm{~d})-2(\mathrm{f})$ shows $\Delta E, \operatorname{Var}(\widehat{H})$, and $\varepsilon$ as a 

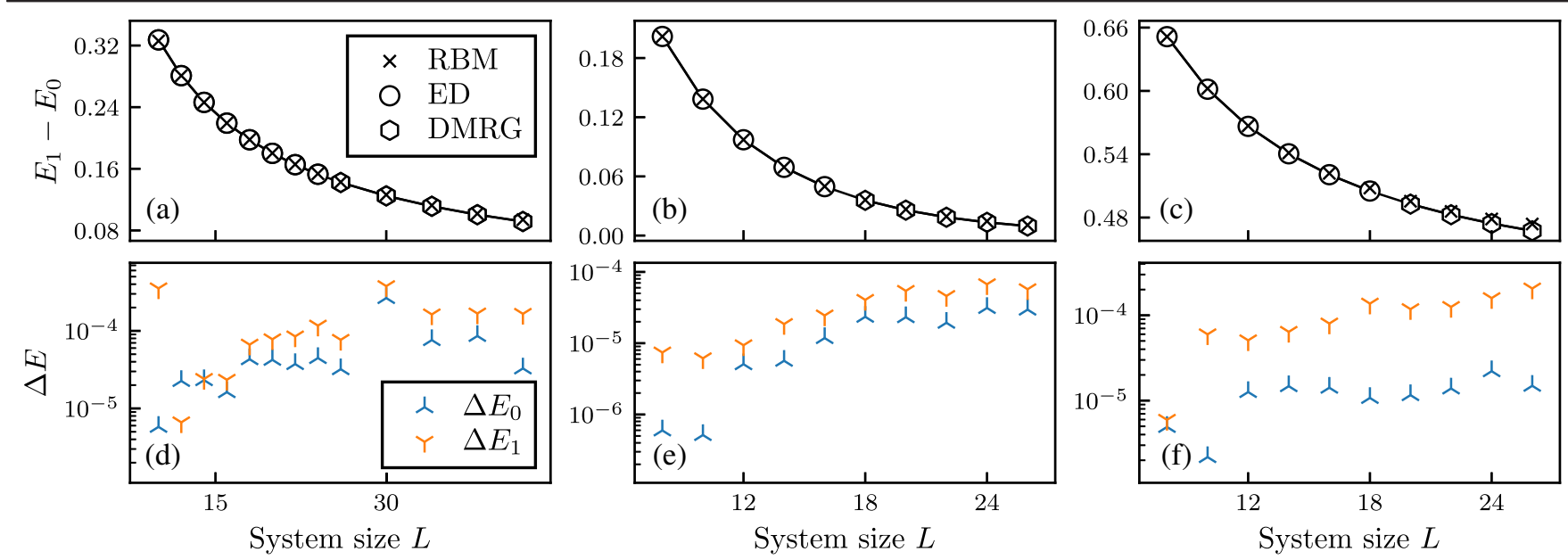

FIG. 3. (a)-(c) Energy gaps $G=E_{1}-E_{0}$ between the first excited state and the ground state for different system sizes. (d)-(f) Relative energy errors for the ground states and the first excited states. The variational energies are compared to those of ED or DMRG. (a), (d) Spin-1/2 AFH ( $\alpha=1)$, (b), (e) spin-1 AFH $(\alpha=2)$, and (c), (f) spin-1 AFH with spin-1/2 edges $(\alpha=2)$. The energies of the DMRG simulations were converged to sufficiently high precision without exploiting SU(2) symmetry.

function of the ratio $\alpha$ in the $s_{z}$ basis and the coupled basis. Across the whole range of $\alpha \in[0.5,4]$, the level of accuracy quantified by these measures is improved by at least an order of magnitude in the coupled basis as compared to the $s_{z}$ basis. These results are indicative for the effectiveness of the coupled basis. The structure of the wave function is similar to that of the spin-1/2 Heisenberg model, and is described in detail in the Supplemental Material [44].

Figure 3(b) shows the energy gap $G$ between the ground state $\left|J=0 M_{J}=0\right\rangle$ and the first excited state $\left|J=1 M_{J}=0\right\rangle$. For the energy gap, excellent agreement is reached between the RBM and ED or DMRG methods for different system sizes. The relative energy error on the ground state is $\mathcal{O}\left(10^{-6}\right)$ for the smallest system sizes, and settles to $\mathcal{O}\left(10^{-5}\right)$ for larger system sizes, while that of the excited state is $\mathcal{O}\left(10^{-5}\right)$ to $\mathcal{O}\left(10^{-4}\right)$. In Fig. 3(c), the energy gap of the spin-1 AFH with physical spin- $1 / 2$ degrees of freedom on the edges is shown. The introduction of spin$1 / 2$ edges lifts the degeneracy of the ground state, and introduces a gap in the system, corresponding to the Haldane gap in the thermodynamic limit. Figure 3(c) shows that the RBM ansatz in the coupled basis can represent gapped systems accurately.

Anyonic golden chain.-As a novel application of the RBM ansatz, we turn to the simulation of anyonic systems. Anyons are defined as degrees of freedom that do not obey the mutual statistics of fermions or bosons [50]. They arise in the fractional quantum Hall effect [51] and play a role in quantum computation $[52,53]$. Here, we study Fibonacci anyons, defined by one anyon type $\tau$ along with the trivial vacuum state 1 . Anyonic many-particle systems are most easily described by fusing the different anyons. For Fibonacci anyons, the fusion rules are given by $\mathbb{1} \otimes \tau=\tau \otimes \mathbb{1}=\tau$ and $\tau \otimes \tau=\mathbb{1} \oplus \tau$. For a one-dimensional system, fusing the $N$ anyons can be done in a linear fashion from left to right [Fig. 1(a)], where $y_{i \in\{1, \ldots, N\}} \equiv \tau$ and $x_{i \in\{1, \ldots, N-1\}} \equiv \mathbb{1}, \tau$. This construction is called a fusion tree. We choose the boundary conditions $x_{0}=x_{N}=\mathbb{1}$. Interacting anyons can be described on the level of the fused anyons. An example is the golden chain, which is reminiscent of the AFH model, defined by the Hamiltonian

$$
\widehat{H}_{\mathrm{GC}}=-\sum_{i=1}^{N-1} \widehat{\mathcal{P}}_{i, i+1}^{\mathbb{1}},
$$

where $\widehat{\mathcal{P}}_{i, i+1}^{\mathbb{1}}$ is the projector on the vacuum fusion channel of the anyons with indices $i$ and $i+1$. The model defined by $\widehat{H}_{\mathrm{GC}}$ is critical.

We exploit RBMs as a variational ansatz to find the ground state of $\widehat{H}_{\mathrm{GC}}$. The results of the intermediate fusings are used as input for the RBM [Fig. 1(b)]. The relative error on the ground-state energy $\Delta E_{0}$, and the variance of the Hamiltonian $\operatorname{Var}\left(\widehat{H}_{\mathrm{GC}}\right)$ are shown in Fig. 4 for different values of $\alpha=M / N$. The relative energy error is below $\mathcal{O}\left(10^{-3}\right)$ for all values of $\alpha$, reaching $\mathcal{O}\left(10^{-5}\right)$ for $\alpha=4$.

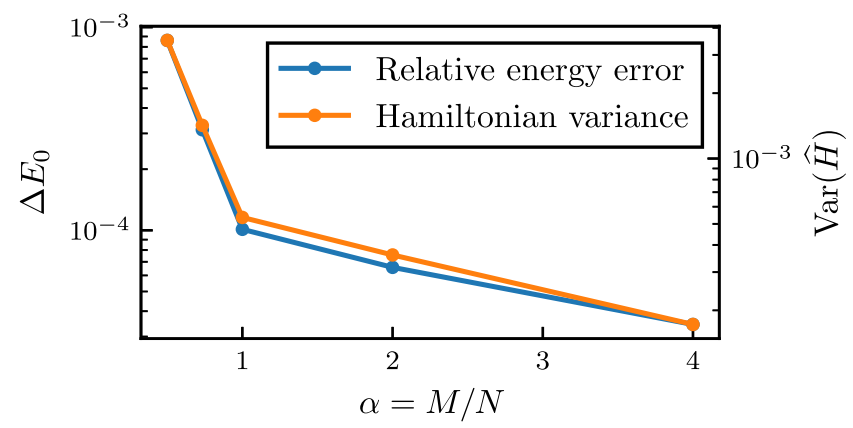

FIG. 4. Relative energy error $\Delta E_{0}$ and variance of the Hamiltonian $\operatorname{Var}\left(\widehat{H}_{\mathrm{GC}}\right)$ of the ground state of the $L=30$ anyonic golden chain. Both measures decrease with the ratio $\alpha$. 
The variance of the Hamiltonian follows the same trend, ranging from $\mathcal{O}\left(10^{-3}\right)$ to $\mathcal{O}\left(10^{-4}\right)$.

Conclusion.-In this Letter, we have extended the variational class of artificial neural network states for spin systems to include non-abelian symmetries, in particular the SU(2) spin-rotation symmetry. Hereto, we have formulated a restricted Boltzmann machine (RBM) ansatz using configurations labeled by intermediate spin coupling quantum numbers. Our numerical findings provide convincing support for using these quantities, which do not depend on the choice of local basis for the spins, as input variables to the RBM. Indeed, our ansatz obtains a higher precision in the ground-state energy for the same amount of variational freedom, compared to previous studies using the basisdependent local spin projections as input variables. In doing so, the states we construct have well-defined total angular momentum $J$ and projection $M_{J}$ quantum numbers and can also be used to target the lowest-lying excited states of the system. Other applications that make use of the specific structure exploited by our ansatz are anyonic chains, for which our ansatz also accurately captures the ground state.

The approach presented here can be used to model spin liquid states, which are invariant under symmetries of the Hamiltonian. Likewise, our method could be adopted for the determination of wave functions in a quantum chemistry context. Both these applications are particularly suitable to be studied with RBMs, due to their potential to model long-range entanglement and correlations, and due to the geometrically independent way of modeling correlations in RBMs. Furthermore, our approach can be generalized for systems in more than one dimension, by defining an appropriate coupling scheme. These include coupling in the form of a graph, such as a tensor network [54], or coupling in a treelike fashion. We stress that our approach is independent of the form of the variational state and is applicable to other variational wave functions.

We thank Stijn De Baerdemacker for useful discussions. We also thank the anonymous referee for suggesting we study correlation functions. The software for this work was built on the NetKet library [55]. The DMRG results were obtained with the ALPS library [56]. The computational resources (Stevin Supercomputer Infrastructure) and services used in this work were provided by the VSC (Flemish Supercomputer Center), and the Flemish Governmentdepartment EWI. This work was supported by Ghent University, Research Foundation Flanders (FWOFlanders), and ERC Grants QUTE (No. 647905) and ERQUAF (No. 715861). T. Vieijra is supported as an "FWO-aspirant" under Contract No. FWO18/ASP/279.

*tom.vieijra@ugent.be

[1] G. Carleo, I. Cirac, K. Cranmer, L. Daudet, M. Schuld, N. Tishby, L. Vogt-Maranto, and L. Zdeborová, Rev. Mod. Phys. 91, 045002 (2019).
[2] J. Carrasquilla and R. G. Melko, Nat. Phys. 13, 431 (2017).

[3] E. P. L. van Nieuwenburg, Y.-H. Liu, and S. D. Huber, Nat. Phys. 13, 435 (2017).

[4] A. Morningstar and R. G. Melko, J. Mach. Learn. Res. 18, 5975 (2018).

[5] M. J. S. Beach, A. Golubeva, and R. G. Melko, Phys. Rev. B 97, 045207 (2018).

[6] K. Ch'ng, J. Carrasquilla, R. G. Melko, and E. Khatami, Phys. Rev. X 7, 031038 (2017).

[7] J. Venderley, V. Khemani, and E.-A. Kim, Phys. Rev. Lett. 120, 257204 (2018).

[8] C. Casert, T. Vieijra, J. Nys, and J. Ryckebusch, Phys. Rev. E 99, 023304 (2019).

[9] Y.-H. Liu and E. P. L. van Nieuwenburg, Phys. Rev. Lett. 120, 176401 (2018).

[10] P. Zhang, H. Shen, and H. Zhai, Phys. Rev. Lett. 120, 066401 (2018).

[11] L. Wang, Phys. Rev. E 96, 051301(R) (2017).

[12] J. Liu, H. Shen, Y. Qi, Z. Y. Meng, and L. Fu, Phys. Rev. B 95, 241104(R) (2017).

[13] J. Liu, Y. Qi, Z. Y. Meng, and L. Fu, Phys. Rev. B 95, 041101(R) (2017).

[14] L. Huang and L. Wang, Phys. Rev. B 95, 035105 (2017).

[15] T. A. Bojesen, Phys. Rev. E 98, 063303 (2018).

[16] G. Torlai and R. G. Melko, Phys. Rev. B 94, 165134 (2016).

[17] Z. Liu, S. P. Rodrigues, and W. Cai, arXiv:1710.04987.

[18] K. Mills and I. Tamblyn, arXiv:1710.08053.

[19] G. Carleo and M. Troyer, Science 355, 602 (2017).

[20] M. Koch-Janusz and Z. Ringel, Nat. Phys. 14, 578 (2018).

[21] S.-H. Li and L. Wang, Phys. Rev. Lett. 121, 260601 (2018).

[22] P. Mehta and D. J. Schwab, arXiv:1410.3831.

[23] S. Iso, S. Shiba, and S. Yokoo, Phys. Rev. E 97, 053304 (2018).

[24] H. Saito, J. Phys. Soc. Jpn. 86, 093001 (2017).

[25] H. Saito and M. Kato, J. Phys. Soc. Jpn. 87, 014001 (2018).

[26] I. Glasser, N. Pancotti, M. August, I. D. Rodriguez, and J. I. Cirac, Phys. Rev. X 8, 011006 (2018).

[27] D.-L. Deng, X. Li, and S. D. Sarma, Phys. Rev. B 96, 195145 (2017).

[28] Y. Zheng, H. He, N. Regnault, and B. A. Bernevig, Phys. Rev. B 99, 155129 (2019).

[29] X. Liang, W.-Y. Liu, P.-Z. Lin, G.-C. Guo, Y.-S. Zhang, and L. He, Phys. Rev. B 98, 104426 (2018).

[30] K. Choo, T. Neupert, and G. Carleo, Phys. Rev. B 100, 125124 (2019).

[31] M. J. Hartmann and G. Carleo, Phys. Rev. Lett. 122, 250502 (2019).

[32] N. Yoshioka and R. Hamazaki, Phys. Rev. B 99, 214306 (2019).

[33] A. Nagy and V. Savona, Phys. Rev. Lett. 122, 250501 (2019).

[34] F. Vicentini, A. Biella, N. Regnault, and C. Ciuti, Phys. Rev. Lett. 122, 250503 (2019).

[35] D.-L. Deng, X. Li, and S. D. Sarma, Phys. Rev. X 7, 021021 (2017).

[36] J. Chen, S. Cheng, H. Xie, L. Wang, and T. Xiang, Phys. Rev. B 97, 085104 (2018).

[37] L. Pastori, R. Kaubruegger, and J. C. Budich, Phys. Rev. B 99, 165123 (2019). 
[38] A. Szabó and N. S. Ostlund, Modern Quantum Chemistry: Introduction to Advanced Electronic Structure Theory (Dover publications, Mineola (N.Y.), 1996).

[39] K. Gunst, F. Verstraete, and D. Van Neck, J. Chem. Theory Comput. 5, 15 (2019).

[40] Y. Zhou, K. Kanoda, and T.-K. Ng, Rev. Mod. Phys. 89, 025003 (2017).

[41] L. Savary and L. Balents, Rep. Prog. Phys. 80, 016502 (2017).

[42] K. Choo, G. Carleo, N. Regnault, and T. Neupert, Phys. Rev. Lett. 121, 167204 (2018).

[43] S. Sorella, Phys. Rev. Lett. 80, 4558 (1998).

[44] See the Supplemental Material at http://link.aps.org/ supplemental/10.1103/PhysRevLett.124.097201 for more information, including technical derivations of the basis transformation, comments on the wave function structure and correlations, and the hyperparameters used in this Letter, which includes Refs. [45-47].

[45] Nuclear Magnetic Resonance of Liquid Crystals, edited by J. Emsly, Nato Science Series C (Springer, Netherlands, 1985).
[46] M. Collura, L. Del'Anna, T. Felser, and S. Montangero, arXiv:1905.11351.

[47] R. H. Swendsen and J.-S. Wang, Phys. Rev. Lett. 57, 2607 (1986).

[48] S. R. White and D. A. Huse, Phys. Rev. B 48, 3844 (1993).

[49] W. Marshall, Proc. R. Soc. Ser. A 232,48 (1955).

[50] S. Trebst, M. Troyer, Z. Wang, and A. W. W. Ludwig, Prog. Theor. Phys. Suppl. 176, 384 (2008).

[51] D. Arovas, J. R. Schrieffer, and F. Wilczek, Phys. Rev. Lett. 53, 722 (1984).

[52] A. Y. Kitaev, Ann. Phys. (N.Y.) 303, 2 (2003).

[53] C. Nayak, S. H. Simon, A. Stern, M. Freedman, and S. D. Sarma, Rev. Mod. Phys. 80, 1083 (2008).

[54] S. Singh and G. Vidal, Phys. Rev. B 86, 195114 (2012).

[55] G. Carleo, K. Choo, D. Hofmann, J. E. T. Smith, T. Westerhout, F. Alet, E. J. Davis, S. Efthymiou, I. Glasser, S.-H. Lin, M. Mauri, G. Mazzola, C. B. Mendl, E. van Nieuwenburg, O. O’Reilly, H. Théveniaut, G. Torlai, and A. Wietek, SoftwareX 10, 100311 (2019).

[56] B. Bauer et al., J. Stat. Mech. (2011) P05001. 